\title{
Hashimoto's encephalitis as a differential diagnosis of Creutzfeldt-Jakob disease
}

M Seipelt, I Zerr, R Nau, B Mollenhauer, S Kropp, B J Steinhoff, C Wilhelm-Gössling, C Bamberg, R W C Janzen, P Berlit, F Manz, K Felgenhauer, S Poser

\begin{abstract}
Department of Neuroradiology, University Hospital, Göttingen, Germany M Seipelt

Department of Neurology, University Hospital, Göttingen, Germany

M Seipelt

I Zerr

$\mathrm{R}$ Nau

B Mollenhauer

S Kropp

B J Steinhoff

K Felgenhauer

$S$ Poser

Department of Neurology, University Medical School, Hanover, Germany C Wilhelm-Gössling

Department of Neurology, Krankenhaus Nordwest Frankfurt am Main, Germany C Bamberg $\mathrm{R}$ W C Janzen

Department of Neurology, Alfried

Abstract

Objectives-During an epidemiological study of Creutzfeldt-Jakob disease in Germany, Hashimoto's encephalitis was encountered as a differential diagnosis, which has not yet been described in this context.

Methods-The symptoms and findings of seven patients who fulfilled the criteria for "possible"Creutzfeldt-Jakob disease are presented.

Results-A Hashimoto's thyroiditis with antibodies against thyroglobulin or thyroid peroxidase, or both and a hypoechoic thyroid ultrasonogram were found in all cases. Analysis of CSF disclosed an increased leucocyte count in three patients, and a raised CSF:serum concentration ratio of albumin (QAlb) in four patients. The 14-3-3 protein, typical of Creutzfeldt-Jakob disease, could not be detected in any of our patients. No periodic sharp wave complexes, which are typical of Creutzfeldt-Jakob disease, were detected on EEG in any of the cases. By contrast with Creutzfeldt-Jakob disease, which leads to death within a few months, the patients with Hashimoto's encephalitis often recover quickly when treated adequately. All the patients improved after administration of corticosteroids. Conclusion-The clinical symptomatology of both diseases may be very similar: dementia, myoclonus, ataxia, and personality change or psychotic phenomena are characteristic symptoms.

(F Neurol Neurosurg Psychiatry 1999;66:172-176)
\end{abstract} Krupp Hospital, Essen, Germany

P Berlit

Department of Neurology, Klinikum

Lippe-Lemgo,

Germany

F Manz

Correspondence to: Dr Maria Seipelt, Klinikum der

Georg-August-Universität, Göttingen, Abteilung für Neuroradiologie, Robert-Koch-Strasse 40, D-37075 Göttingen, Germany. Telephone 0049 55139 6636; fax 0049551 397020 .

Received 11 May 1998 and in revised form 25 August 1998

Accepted 11 September 1998
Keywords: Hashimoto's encephalitis; Hashimoto's thyroiditis; Creutzfeldt-Jakob disease; encephalopathy

In the course of our epidemiological study of Creutzfeldt-Jakob disease in Germany, we encountered Hashimoto's encephalitis as a differential diagnosis, which has not yet been described in this context. ${ }^{1}$

Hashimoto's encephalitis is associated with Hashimoto's thyroiditis. ${ }^{2}$ As yet, no data are available on the incidence of this disease; it is much more often found in women than in men. The most common symptoms are myoclonus, epileptic seizures, dementia, and disturbances of consciousness. In most cases therapy with corticosteroids leads to clear improvement or even disappearance of the symptoms.
Chronic lymphocytic Hashimoto's thyroiditis is an often occurring $(3 \%-4 \%$ of the population) organ specific autoimmune disease accompanied by development of goitre. ${ }^{29}$ Middle aged women are most often affected. About one third of the patients are hypothyroid. In almost all cases antibodies against thyroglobulin, or thyroid peroxidase, or both are detectable. ${ }^{30-32}$ Another typical feature is a hypoechoic thyroid ultrasonogram. Fine needle biopsy discloses inflammatory infiltrates with lymphocytes, plasma cells, colloid accumulation, and cell detritus.

Creutzfeldt-Jakob disease is characterised by rapidly progressive dementia, myoclonus, pyramidal and extrapyramidal signs, and cerebellar symptoms. ${ }^{3}$ It is a rare disease (with an incidence of about one case/1000 000 population/ year), usually occurring in persons aged $60-70$ years. Creutzfeldt-Jakob disease is also more often seen in women than in men. In most cases death occurs within a few months after onset. The clinical suspicion is corroborated by EEG and by analysis of CSF. ${ }^{4-9}$ An effective treatment for the disease is not available.

The clinical symptomatology of Hashimoto's encephalitis and Creutzfeldt-Jakob disease may be very similar. Above all, dementia, myoclonus, ataxia, and personality change or psychotic phenomena are characteristic symptoms of these two diseases (table 1).

We could not find any data on the frequency with which encephalitis occurs in pre-existing Hashimoto's thyroiditis. Also, no information is available on the number of cases in which cerebral symptoms of unclear origin with myoclonus, dementia, and further neurological symptoms are associated with Hashimoto's thyroiditis.

To our knowledge, 46 cases have been reported that were mainly published as case reports in 20 publications. $^{2}{ }^{10-28}$

It is still unclear whether the cerebral manifestation of Hashimoto's thyroiditis represents an autoimmune reaction against a shared antigen or whether a cerebral autoimmune vasculitis is merely associated with autoimmune thyroiditis. The pleocytosis in the CSF of some of our patients suggests the presence of chronic inflammation. We therefore used the term "encephalitis" throughout this publication. However, we were unable to show intrathecal synthesis of antibodies against thyroid antigens. 
Table 1 Symptoms of Creutzfeldt-Fakob disease and Hashimoto's encephalitis

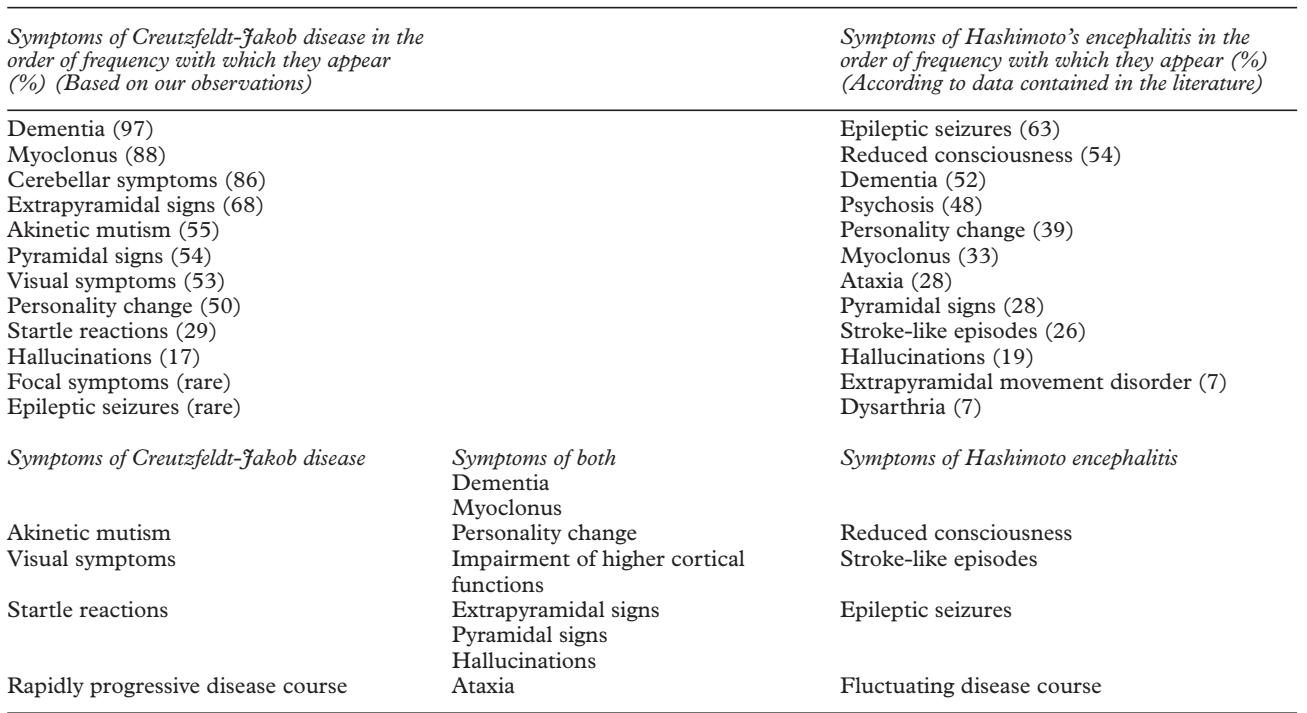

Hashimoto's encephalitis is presented here as a differential diagnosis of Creutzfeldt-Jakob disease.

\section{Methods}

Since June 1993, the Creutzfeldt-Jakob research unit of the University of Göttingen has examined all patients throughout Germany, who were notified as suspected cases of Creutzfeldt-Jakob disease to this unit. According to the criteria of Masters et al (modified according to Will) the suspected cases are, in conformity with the European BIOMED 1 study, classified as "possible", "probable" and "definite" cases or as "others". 33 34

The following cases with Hashimoto's encephalitis were initially classified as "possible" cases of Creutzfeldt-Jakob disease. Based on these cases, the clinical picture of Hashimoto's encephalitis was studied.

\section{Results}

The symptoms and findings for the seven patients presented here are summarised in table 2. At disease onset the diagnosis of Creutzfeldt-Jakob disease was suspected in the first female patient (case 1). The correct diagnosis of Hashimoto's encephalitis was established later in the course of the disease. The administration of corticosteroids resulted in a rapid recovery of the patient. The disease course of this patient attracted our attention and, therefore, thyroid antibodies were also determined in other patients suspected to have Creutzfeldt-Jakob disease. As a result of these laboratory findings and of a hypoechoic thyroid ultrasonogram, Hashimoto's encephalitis was diagnosed in four other patients (cases 2, 3, 4, and 5). Case histories of two patients with the diagnosis of Hashimoto's encephalitis were included, who would have fulfilled the criteria

Table 2 Summary of symptoms and findings in our patients with Hashimoto's encephalitis

\begin{tabular}{|c|c|c|c|c|c|c|c|}
\hline Case & 1 & 2 & 3 & 4 & 5 & 6 & 7 \\
\hline Age & 58 & 54 & 44 & 53 & 73 & 80 & 86 \\
\hline Sex & $\mathrm{F}$ & $\mathrm{F}$ & $\mathrm{F}$ & $\mathrm{F}$ & $\mathrm{F}$ & $\mathrm{F}$ & $\mathrm{F}$ \\
\hline \multicolumn{8}{|l|}{ Symptoms: } \\
\hline Dementia & + & + & + & + & + & + & + \\
\hline Cerebellar ataxia & + & + & + & + & + & + & + \\
\hline Myoclonus & + & + & + & + & + & + & + \\
\hline Extrapyramidal signs & & & & + & & & \\
\hline Pyramidal signs & + & & & & & & + \\
\hline Hallucinations & + & & & + & + & & \\
\hline Reduced consciousness & + & + & & + & + & + & \\
\hline Epileptic seizures & + & + & & & & & \\
\hline Psychosis & & + & + & & + & & \\
\hline Stroke-like episodes & & & & + & + & + & + \\
\hline Apathy & + & + & + & & + & & + \\
\hline \multicolumn{8}{|l|}{ Laboratory findings: ${ }^{\star}$} \\
\hline Thyroid parameters & Euthyroid & Hyperthyroid & Euthyroid & Euthyroid & Euthyroid & Euthyroid & Euthyroid \\
\hline TSH & Increased & Reduced & Normal & Normal & Normal & Normal & Increased \\
\hline Thyroglobin antibodies & $232 \mathrm{U} / \mathrm{ml}$ & $147 \mathrm{U} / \mathrm{ml}$ & Normal & $171 \mathrm{U} / \mathrm{ml}$ & Normal & $\dagger$ & Normal \\
\hline $\begin{array}{l}\text { Microsomal antibodies } \\
\text { CSF findings: }\end{array}$ & Normal & Normal & $548 \mathrm{U} / \mathrm{ml}$ & Normal & $205 \mathrm{U} / \mathrm{ml}$ & $t$ & $1602 \mathrm{U} / \mathrm{ml}$ \\
\hline Leucocyte density & 13 leuc/ $\mu \mathrm{l}$ & 21 leuc/ $\mu 1$ & Normal & 5 leuc/ $\mu \mathrm{l}$ & Normal & Normal & Normal \\
\hline Protein in CSF & Increased & Increased & Increased & Increased & Normal & Normal & Increased \\
\hline $\mathrm{CSF} /$ serum albumin ratio & Increased & Increased & Increased & Increased & Normal & Normal & Increased \\
\hline Brain atrophy: & No & No & Yes & No & Yes & No & Yes \\
\hline
\end{tabular}

*The following thyroid parameters were determined: triiodothyronine, free L-thyroxin, thyroid-stimulating hormone (TSH), thyroglobulin antibodies (normal range $<60 \mathrm{U} / \mathrm{ml}$ ), microsomal antibodies (=autoantibodies to thyroid peroxidase, normal range $<60 \mathrm{U} / \mathrm{ml}$ ).

†In this case Hashimoto's encephalitis was diagnosed because of neurological symptoms, excluding other diseases and improvement after corticoid therapy. 
for "possible" Creutzfeldt-Jakob disease (cases 6 and 7). In the following, three representative cases are reported.

CASE 1

Beginning in May 1997, a 58 year old woman developed memory disorders, dysarthria, vertigo, and an increasingly unsteady gait. In June 1997 she experienced a grand mal seizure. In the subsequent weeks, she developed a progressive organic brain syndrome accompanied by impaired cognitive abilities, optical hallucinations, aggressiveness, and progressive dementia; the patient reacted slowly, showed startle reactions, and was intermittently somnolent. Neurological examinations disclosed increasing static and truncal ataxia. Left sided generalised myoclonus occurred. In July 1997 an obvious lack of initiative was noted in the patient and she was confined to bed; the diagnosis of "possible" Creutzfeldt-Jakob disease was made. In August 1997 she finally became stuporos, had a series of epileptic seizures, and showed right sided positive pyramidal signs. In September 1997 the diagnosis of Hashimoto's encephalitis was established. The patient had been treated with L-thyroxin for Hashimoto's thyroiditis for 10 years.

Four days after starting corticosteroid therapy she became more vigilant, started to speak, showed reorientation to person and place, and increasingly performed activities of daily living on her own. Myoclonus stopped after giving valproate. Impaired cognitive abilities and disorientation to time persisted.

CASE 2

This 57 year old woman fell ill in August 1994. She had vertigo, impaired cognitive abilities, tremor, headache, and increasing gait disturbances and became somnolent. Three weeks later she additionally showed paranoia, aggressive behaviour, and psychomotor restlessness. Predominantly right sided generalised myoclonus occurred. The patient became comatose, and developed generalised epileptic seizures, hypertension, and fever. In the course of the disease, symptoms were fluctuating, especially the state of consciousness. At the beginning of 1995 she deteriorated dramatically; symptoms included psychotic episodes, generalised myoclonus, and the clinical picture of akinetic mutism. The patient was notified as a suspected case of Creutzfeldt-Jakob disease and classified as "possible" case.

The administration of dexamethasone quickly improved the patient's symptoms. After a vertebral fracture, treatment was discontinued; again, her condition deteriorated. Treatment with immunosuppressants, initially with azathioprine, later with methotrexate ( $5 \mathrm{mg} /$ week), relieved her symptoms but with fluctuations until now.

CASE 3

Since July 1996, a 44 year old woman had increasingly impaired cognitive abilities, memory disorders, and depressive mood. In February 1997, she was admitted to a psychiatric hospital due to a paranoid psychosis. Myo- clonus in all limbs occurred and appeared irregularly several times a minute. After administration of clonazepam and valproate the myoclonus disappeared. In June 1997, lack of initiative and blunted affect were noted. Tests of standing and walking showed unsteadiness.

Corticosteroid therapy resulted in gradual improvement of the symptomatology. Treatment with clonazepam and valproate was discontinued and myoclonus did not recur.

In the seven patients of this study the initial symptomatology varied. Personality change, confusion, psychotic episodes, dementia, myoclonus, epileptic seizures, and disturbances of consciousness usually developed within a few weeks. The determination of the thyroid autoantibodies in serum is important; the thyroglobulin antibodies or the microsomal antibodies were raised in all cases. In one case increased titres of TSH receptor antibodies were additionally found. The other laboratory indices were less reliable. Four patients were euthyroid, two patients were subclinically hypothyroid, and one patient was hyperthyroid. In six patients, Hashimoto's thyroiditis had not been diagnosed before.

The analysis of CSF disclosed several abnormalities. In three patients the leucocyte density was increased, in five patients the CSF:serum concentration ratio of albumin (Qalb) was raised. However, we were unable to show intrathecal synthesis of antibodies against thyroid antigens. Likewise, isoelectric focusing showed no oligoclonal bands in CSF. The 14-3-3 protein, typical of Creutzfeldt-Jakob disease, could not be detected in any of these patients.

Three cases did not show any abnormalities on cranial CT. Slight brain atrophy was detected in three cases, and a meningioma of the sphenoid bone as a secondary finding in one case. Brain MRI also did not show any specific findings. Apart from brain atrophy, leukoencephalopathic changes were seen in one patient. One patient had lacunar infarctions, another patient had a meningioma of sphenoid bone previously detected by CT.

Examinations by EEG showed non-specific generalised or lateralised abnormalities in all patients; foci were found in two patients. No periodic sharp wave complexes, which are typical of Creutzfeldt-Jakob disease, were detected in these patients.

After corticosteroid therapy, the clinical picture of all patients clearly improved. The symptoms of two patients disappeared, three patients showed residual symptoms, and two patients showed a fluctuating disease course.

\section{Conclusion}

Hashimoto's encephalitis is a rare disease that is associated with Hashimoto's thyroiditis. Hashimoto's thyroiditis is suspected when thyroid autoantibodies in serum are raised; the definite diagnosis is based on sonography or fine needle biopsy. As yet, no data are available on the incidence of Hashimoto's encephalitis. The mean age of the patients reported previously was 47 years (range 14-78), $85 \%$ of 
the patients were female; all seven patients presented by us are women with a mean age of 64 years (range 44-86 years). The neurological symptoms of Hashimoto's encephalitis are not specific. Unfortunately, little information is available on the early manifestations of this disease. Our patients are biased as they became known in the Creutzfeldt-Jakob disease research programme. For this reason, symptoms such as dementia, myoclonus, and ataxia that are typical of Creutzfeldt-Jakob disease were observed more often in our patients, whereas epileptic seizures and psychoses appeared less often in this series than in those cases hitherto published.

Limited data are available on the analysis of CSF in this disease. About $65 \%$ of the published cases, in accordance with our findings, showed an increase in the protein concentration in CSF (between 400 and 1800 $\mathrm{mg} / \mathrm{l}) ; 71 \%$ of our patients had a raised CSF:serum concentration ratio of albumin $\left(\mathrm{Q}_{\mathrm{Alb}}\right.$ 8-24; no previous data available). However, it remains to be determined whether this is due to reduction of CSF turnover in brain atrophy or increased protein permeability of capillaries adjacent to the CSF space. Two of our five patients with a raised $\mathrm{Q}_{\mathrm{Alb}}$ had brain atrophy; one of the three patients with brain atrophy had no increase in $\mathrm{Q}_{\mathrm{Alb}}$. The leucocyte density in the CSF of three of our patients $\left(5,13\right.$, and 21 leucocytes $\left./ \mathrm{mm}^{3}\right)$ and in $15 \%$ of the published cases (maximum 169 leucocytes/ $\mathrm{mm}^{3}$ ) was increased. The pleocytosis in the CSF suggests the presence of chronic inflammation. Unfortunately we were not able to obtain reliable CSF:serum concentration ratios of thyroid antibodies. Isoelectric focusing showed no oligoclonal bands in the CSF. The 14-3-3 protein was not detected in the CSF of any of our patients. This protein is typically found in the CSF of patients with CreutzfeldtJakob disease. ${ }^{67}$ In conformity with the cases reported previously, we found slow background activity in EEG examinations, but no periodic sharp wave complexes which are characteristic of Creutzfeldt-Jakob disease. ${ }^{4}$ The neuroradiological findings were also nonspecific: three patients showed generalised brain atrophy and one patient also showed leukoencephalopathic changes. Further studies will show whether this finding of reversible oedema in the white matter turns out to be important for the diagnosis or not. ${ }^{24}$

The pathogenesis of Hashimoto's encephalitis is unclear. Autoimmune vasculitis, possibly with deposition of immune complexes, reversible leukoencephalopathy with surrounding oedema, or the presence of a shared antigen in the thyroid gland and in the brain have been discussed. ${ }^{2} 121415242835$ An excessive, central release of TRH was held responsible for the epileptic seizures. ${ }^{14}$

In the diagnosis of rapidly progressive dementia, Hashimoto's encephalitis should be considered as an important differential diagnosis. ${ }^{25}$ By contrast with Creutzfeldt-Jakob disease, which leads to death within a few months, the patients with Hashimoto's encephalitis often recover quickly when treated adequately. Corticosteroid therapy is the first choice. The successful administration of other immunosuppressants (methotrexate, azathioprine, cyclophosphamide) has been reported (see case 2). ${ }^{10}{ }^{16}$ Epileptic seizures and myoclonus usually respond well to anticonvulsant drugs. Even with consistent therapy relapsing disease courses are not rare, but the patients altogether clearly benefit from this therapy.

In cases of unclear encephalopathy with dementia, myoclonus, epileptic seizures, and other neurological symptoms, the thyroid autoantibodies should be determined and corticosteroid therapy should be instituted if they are raised. In $10 \%$ of the normal population, however, positive thyroid autoantibodies are present. ${ }^{16}$ The diagnosis of Hashimoto's thyroiditis is established by the detection of clinical or subclinical hypothyroidism and a hypoechoic ultrasonographic pattern of the thyroid gland. In doubtful cases, the diagnosis is established by fine needle biopsy. The response to corticosteroid therapy, the absence of 14-3-3 protein and periodic sharp wave complexes support the diagnosis of Hashimoto's encephalitis.

Probably Hashimoto's encephalitis is underdiagnosed. In elderly people, the most important differential diagnosis to Creutzfeldt-Jakob disease remains rapidly progressive Alzheimer's disease. In younger subjects, however, chronic encephalitis, and as a subgroup Hashimoto's encephalitis, is the most frequent disease which has to be excluded in suspected CJD cases.

All of our patients of the present series had severe disease courses of Hashimoto's encephalitis, which suggested the diagnosis of Creutzfeldt-Jakob disease. In the literature, too, predominance of severe disease courses is documented. The clinical appearance of early Hashimoto's encephalitis is unknown. Together with the outpatient clinic for thyroid diseases, a prospective study has been set up to examine early neurological symptoms in patients with Hashimoto's thyroiditis.

This study was supported by a grant from the Bundesministerium für Gesundheit to HAK and to SP (BMG Az325-447305/3). We are grateful to Inge Schreivogel, Johannes Meller, and Professor Wolfgang Becker from the Department of Nuclear Medicine of the University Hospital of Göttingen for their help in diagnosis and treatment of Hashimoto's thyroiditis. We thank in diagnosis and treatment of Hashimoto's thyroiditis. We thank Professor E König from the Department of Neurology in the Hospital Bad Aibling for the clinical data of a patient with Hashimoto's encephalitis. We acknowledge the work of the site visiting physicians Kati Weidehaas, Anke Otto, Markus Otto, and Holger Schmidt and thank Monika Bodemer for analysis of CSF. We thank all physicians notifying suspect cases to the German Creutzfeldt-Jakob disease surveillance unit, for providing clinical, neuroradiological, and biochemical data, as well as for their help in obtaining CSF specimens. Special thanks go to Antje Isbrandt, Maja Schneider-Dominco, Markus Otto, and Bernhard Haug for their support.

1 Poser S, Zerr I, Schulz-Schaeffer WJ, et al. Die CreutzfeldtJakob-Krankheit. Eine Sphinx der heutigen Neurobiologie. Dtsch Med Wochenschr 1997;122:1099-105.

2 Brain L, Jellinek EH, Ball K. Hashimoto's disease and encephalopathy. Lancet 1966;ii:512-4.

3 Brown P, Gibbs CJ, Rodgers-Johnson P, et al. Human pongiform encephalopathy: the National Institutes of Health series of 300 cases of experimentally transmitted disease. Ann Neurol 1994;35:513-29.

4 Steinhoff BJ, Racker S, Herrendorf G, et al. Accuracy and reliability of periodic sharp wave complexes in Creutzfeldtreliability of periodic sharp wave complexes

5 Otto M, Stein H, Szudra A, et al. S-100 protein concentration in the cerebrospinal fluid of patients with CreutzfeldtJakob disease. $\mathcal{F}$ Neurol 1997;244:566-70. 
6 Otto M, Wiltfang J, Tumani H, et al. Elevated levels of tauprotein in cerebrospinal fluid of patients with Creutzfeldtprotein in cerebrospinal fluid of patients with
Jakob disease. Neurosci Lett 1997;225:210-2.

7 Hsich G, Kenney K, Gibbs CJ, et al. The 14-3-3 brain protein in cerebrospinal fluid as a marker for transmissible spongiform encephalopathies [comments]. $N$ Engl $\mathcal{F ~ M e d ~}$ 1996;335:924-30.

8 Zerr I, Bodemer M, Gefeller O, et al. Detection of 14-3-3 protein in the cerebrospinal fluid supports the diagnosis of Creutzfeldt-Jakob Disease. Ann Neurol 1998;43:32-40.

9 Zerr I, Bodemer M, Racker S, et al. Cerebrospinal fluid concentration of neuron-specific enolase in diagnosis

10 Kruse H. Thyrotropin-releasing hormone: interaction with chlorpromazine in mice, rats, and rabbits. 7 Pharmacol 1975;6:249-68

11 Brown M, Vale W. Central nervous system effects of hypothalamic peptides. Endocrinology 1975;96:1333-6.

12 Thrush DC, Boddie HG. Episodic encephalopathy associated with thyroid disorders. $\mathcal{F}$ Neurol Neurosurg Psychiatry 1974:37:696-700.

13 Jellinek EH, Ball K. Letter: Hashimoto's disease, encephalopathy, and splenic atrophy. Lancet 1976;i:1248.

14 Latinville D, Bernardi O, Cougoule JP, et al. Hashimoto's thyroiditis and myoclonic encephalopathy. Pathogenic hypothesis. Rev Neurol (Paris) 1985;141:55-8.

15 Shein M, Apter A, Dickerman Z, et al. Encephalopathy in compensated Hashimoto thyroiditis: a clinical expression
of autoimmune cerebral vasculitis. Brain Dev 1986;8:60-4.

16 Shaw PJ, Walls TJ, Newman PK, et al. Hashimoto's encephalopathy: a steroid-responsive disorder associated with high anti-thyroid antibody titers; report of 5 cases. Neurology 1991;41:228-33.

17 Ghawche F, Bordet R, Destee A. Hashimoto's encephalopathy: toxic or autoimmune mechanism? Rev Neurol (Paris) 1992;148:371-3.

18 Takahashi S, Mitamura R, Itoh Y, et al. Hashimoto encephalopathy: etiologic considerations. Pediatr Neurol 1994;11:328-31.

19 Henchey R, Cibula J, Helveston W, et al. Electroencephalographic findings in Hashimoto's encephalopathy. Neurology 1995;45:977-81.

20 Ishii K, Hayashi A, Tamaoka A, et al. Case report: thyrotropin-releasing hormone-induced myoclonus and tremor in a patient with Hashimoto's encephalopathy. $A m \mathcal{F}$ Med Sci 1995;310(5):202-5.
21 Bostantiopoulou S, Zafiriou D, Katsarou Z, Kazis A. Hashimoto's encephalopathy: clinical and laboratory findings.

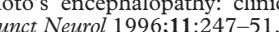

22 Ghika-Schmid F, Ghika J, Regli F, et al. Hashimoto's myoclonic encephalopathy: an underdiagnosed treatable condition [comments]? Mov Disord 1996;11:555-62.

23 Kothbauer-Margreiter I, Sturzenegger M, Komor J, et al. Encephalopathy associated with Hashimoto thyroiditis: diagnosis and treatment. F Neurol 1996;243:585-93.

24 Bohnen NI, Parnell KJ, Harper CM. Reversible MRI findings in a patient with Hashimoto's encephalopathy. Neurology 1997;49:246-7.

25 Forchetti CM, Katsamakis G, Garron DC. Autoimmune thyroiditis and a rapidly progressive dementia: global hypoperfusion on SPECT scanning suggests a possible mechanism. Neurology 1997;49:623-6.

26 Maeda K, Tanimoto K. Epileptic seizures induces by thyrotropin releasing hormone. Lancet 1981;i:1058-9.

27 Hepin releasing hormone. Lancet 1981;1:1058-9. encephalopathy: a new neuroimmunological syndrome. Ann Neurol $1987 ; 22: 140-1$.

28 Mauriac L, Roger P, Kern AM, et al. Thyroidite de Hashimoto et encéphalopathie. Rev Franc Endocrinol Clin Hashimoto et encer

29 Flynn SD, Nishiyama RH, Bigos ST. Autoimmune thyroid disease: immunological, pathological, and clinical aspects. Crit Rev Clin Lab Sci 1988;26:43-95.

30 Mariotti S, Ruf J, Caturegli P, et al. Methodological approach and diagnostic usefulness of a new assay for antithyroid peroxidase autoantibodies. Ann Biol Clin (Paris) 1989;47:541-5.

31 Mariotti S, Chiovato L, Vitti P, et al. Recent advances in the understanding of humoral and cellular mechanisms implicated in thyroid autoimmune disorders. Clin Immunol Immunopathol 1989;50:S73-84.

32 Jordan MW. Hashimoto's thyroiditis. F Am Osteopath Assoc 1967;66:1262-9.

33 Masters CL, Harris JO, Gajdusek DC, et al. CreutzfeldtJakob disease: patterns of worldwide occurrence and the significance of familial and sporadic clustering. Ann Neurol $1979 \cdot 5: 177-88$.

34 Will RG. The spongiform encephalopathies [editorial]. $f$ Neurol Neurosurg Psychiatry 1991;54:761-3.

35 Shibata N, Yamamoto Y, Sunami N, et al. Isolated angiitis of the CNS associated with Hashimoto's disease. Rinsho Shinkeigaku 1992;32:191-8. 